\title{
REVISIÓN DE MATTFELDANTHUS, UN GÉNERO DE VERNONIEAE (ASTERACEAE) ENDÉMICO DEL NORDESTE DE BRASIL
}

\author{
MASSIMILIANO DEMATTEIS ${ }^{1,2}$
}

\begin{abstract}
Summary: Dematteis, M. 2005. Revision of Mattfeldanthus, a genus of Vernonieae (Asteraceae) endemic to northeastern Brazil. Bonplandia 14(1-2): 73-81. ISSN: 0524-0476.

Mattfeldanthus H. Rob. \& R. M. King constitutes one of the few South American genera of Vernonieae with zygomorphic corollas. It has two species endemic to northeastern Brazil that occur in "caatinga" and "campo rupestre" vegetation. The species can be distinguished from each other by several morphological features including the shape of their involucres, capitulum number, floret size and phyllary shape. In this paper the two species of Mattfeldanthus are described and illustrated. In addition, the phylogenetic relationships of the genus to other in Vernonieae are discussed and the geographic distribution of the species is revised to include a larger area of northeastern Brazil.
\end{abstract}

Key words: taxonomy, Vernonieae, Mattfeldanthus, Brazil.

Resumen: Dematteis, M. 2005. Revisión de Mattfeldanthus, un género de Vernonieae (Asteraceae) endémico del nordeste de Brasil. Bonplandia 14(1-2): 73-81. ISSN: 0524-0476.

Mattfeldanthus H. Rob. \& R. M. King constituye uno de los pocos géneros de la tribu Vernonieae con corolas cigomorfas. Este grupo tiene dos especies endémicas del nordeste de Brasil que habitan en la "caatinga" y en campos rupestres. Las especies se pueden diferenciar entre ellas por varios caracteres morfológicos tales como la forma de sus involucros, el número de capítulos, el tamaño de las flores y la forma de los filarios. En el presente trabajo se describen e ilustran las dos especies de Mattfeldanthus. También se discuten las relaciones filogenéticas del género con otras Vernonieae y se amplía la distribución geográfica de las especies a un área mayor del nordeste de Brasil.

Palabras clave: taxonomía, Vernonieae, Mattfeldanthus, Brasil.

\section{Introducción}

La tribu Vernonieae Cass. (Asteraceae) se encuentra ampliamente distribuida en las regiones tropicales y subtropicales de Asia, África y América, pero está especialmente re- presentada en Sudamérica, donde habitan alrededor de 600 especies concentradas principalmente en Brasil, Argentina, Paraguay y Bolivia (Bremer, 1994).

Actualmente la tribu está dividida en diez subtribus, que se diferencian entre sí por el tipo de polen, número de flores, receptáculo,

1 Instituto de Botánica del Nordeste (UNNE-CONICET), C.C. 209, 3400, Corrientes, Argentina. E-mail: dematteisar@yahoo.com.ar

${ }^{2}$ Miembro de la carrera del Investigador Científico del CONICET 
compuestos químicos y número cromosómico (Robinson, 1999). La subtribu Vernoniinae Less. constituye el grupo sudamericano más grande, con alrededor de 25 géneros y más de 450 especies. La mayor parte de las especies de la subtribu pertenecen al extenso género Vernonia Schreb., para el cual se han propuesto distintas clasificaciones infragenéricas (Bentham, 1873; Baker, 1873; Cabrera, 1944; Keeley, 1978; Jones, 1979, 1981; Keeley \& Turner, 1990).

En el tratamiento más reciente de la tribu, Robinson (1999) segrega casi todas las especies sudamericanas de Vernonia a nuevos géneros. Sin embargo, a pesar de la reorganización efectuada por este autor, la identidad y posición de muchas especies siguen siendo prácticamente desconocidas (Hind, 1993; Robinson, 1988, 1999; Dematteis, 2003). Esto ocurre especialmente con las Vernonieae de Brasil, país con escasas revisiones de géneros y muchas entidades conocidas solamente por sus ejemplares tipo o en algunos casos por los fototipos.

Uno de los grupos poco estudiados es Mattfeldanthus H. Rob. \& R. M. King, el cual no ha sido analizado exhaustivamente luego de su descripción (Robinson \& King, 1979). Este género se estableció inicialmente para incluir a una especie atípica de Vernonieae endémica de Bahía, en el nordeste de Brasil. Esta especie se caracteriza por presentar corolas cigomorfas en las flores periféricas de los capítulos, a diferencia de la mayoría de las especies americanas de la tribu que muestran siempre corolas actinomorfas. Las flores marginales de dicha especie presentan una incisión más corta en los cuatro lóbulos externos de la corola, mientras que el lóbulo interno muestra una incisión profunda, similar a las corolas actinomorfas de las flores centrales.

El mismo año en que se describió el género, Robinson (1979) dio a conocer una nueva especie de Vernonia, que presenta algunos caracteres comunes a Mattfeldanthus. Sin embargo, todas las flores de la nueva entidad muestran un lóbulo más largo que los restantes, debido a lo cual el autor incluyó esta especie en Vernonia. Posteriormente, el mismo Robinson (1980) transfirió dicha especie a Mattfeldanthus, valorizando la presencia de corolas cigomorfas.

Con anterioridad a la descripción de esta especie, Barroso (1960-1961) describe una especie de Vernonia basándose en material coleccionado en Pernambuco, la cual presenta el mismo tipo de ramificación e involucro que Mattfeldanthus. Sin embargo, en el protólogo no se hizo referencia a la morfología de las flores de la especie, por lo que ésta permaneció en una posición incierta durante algún tiempo. El estudio del material típico y ejemplares adicionales de la especie realizado por Dematteis (2003), muestra que la misma presenta todas las flores cigomorfas, por lo cual se transfirió esta especie a Mattfeldanthus, pasando la especie descripta por Robinson (1979) a su sinonimia.

Actualmente el género cuenta con dos especies, que tienen como rasgo distintivo la presencia de flores con corola cigomorfa en la periferia o en todo el capítulo. Este carácter no es exclusivo de Mattfeldanthus, ya que existen otros géneros de Vernonieae con corola cigomorfa. Este es un carácter común de los tres géneros de la subtribu Elephantopodinae Less.: Elephantopus L., Pseudelephantopus Rohr y Orthopappus Gleason. Sin embargo, en esta subtribu solamente una de las incisiones de la corola es más profunda que las restantes y los cinco lóbulos están orientados hacia el mismo lado, dispuestos en forma paralela. Debido a la morfología y orientación de los lóbulos, este tipo de corola ha sido descripto tradicionalmente como palmada (Lessing, 1829; De Candolle, 1836) o subpalmada (Baker, 1873).

Otro género americano de la tribu con flores cigomorfas es Stokesia L'Hér., del sudeste de Estados Unidos. Pero a diferencia de los géneros anteriores, Stokesia presenta flores marginales liguladas y flores centrales tubulosas, 5-lobadas. Por el tipo de flores que presenta, la posición de Stokesia en la tribu es aún motivo de controversia (Jones, 1977; Jeffrey, 1988; Robinson, 1999). Esta discusión se sustenta también en datos cromosómicos, ya que Stokesia presenta número básico $\mathrm{x}=7$, lo cual la distingue claramente de las restantes Vernonieae que tienen siempre números mayores a $\mathrm{x}=9$ (Keeley $\&$ Turner, 1990; Dematteis, 2002). 
En la subtribu Vernoniinae Less., el único género que presenta corolas cigomorfas es Dipterocypsela S. F. Blake, un género monotípico endémico de Colombia (Blake, 1945). En este caso, todas las corolas presentan dos lóbulos de mayor tamaño que los restantes, los cuales están orientados hacia la parte externa del capítulo. Dipterocypsela se diferencia además de Mattfeldanthus por el papus que es pluriseriado y los aquenios alados y notablemente comprimidos, características excepcionales en la tribu.

Además del tipo de flores, Mattfeldanthus se puede distinguir también por el tipo de ramificaciones, la forma del involucro y la morfología de los granos de polen. Las ramificaciones principales de la inflorescencia de Mattfeldanthus generalmente son opuestas y notablemente flexuosas, lo cual no es común en las Vernonieae, que muestran por lo general ramificaciones alternas y rectas.

El involucro de Mattfeldanthus tiene forma acampanada o cilíndrico-acampanada, como la mayoría de las Vernonieae, pero está formado por 8-10 series de filarios. Ese número de series de filarios se presenta en algunas especies de Vernonia subsect. Macrocephalae Benth. (=Lessingianthus H. Rob.) afines a $V$. sessilifolia Less., tales como $V$. ramellae $\mathrm{Ca}-$ brera y $V$. bellula Dematteis. Sin embargo, a diferencia de Mattfeldanthus, las especies de este grupo presentan involucro hemisférico y capítulos largamente pedunculados.

Los granos de polen de Mattfeldanthus son equinolofados, tricolporados, con muros discontinuos, microperforados y lagunas dispuestas regularmente, sin una laguna polar. Este tipo de grano ha sido denominado tipo "G" por Robinson (1988) y en Sudamérica se encuentra presente también en Xiphochaeta Poepp. y algunas especies de Stilpnopappus Mart. ex DC. y Lepidaploa Cass. (= Vernonia subsect. Axilliflorae Benth.). Este último constituye el grupo más cercano a Mattfeldanthus, pero difiere de éste por presentar flores actinomorfas y diferente tipo de polen, entre otros caracteres.

Las dos especies con que cuenta el género son relativamente poco frecuentes y muestran además una distribución geográfica restringida, por lo cual el conocimiento de las mismas es relativamente limitado. En el presente trabajo se describen e ilustran las dos especies de Mattfeldanthus reconocidas hasta ahora y se presenta una clave para la identificación de las mismas.

\section{Mattfeldanthus H. Rob. \& R. M. King}

Robinson, H. \& R. M. King, Willdenowia 9: 10. 1979. Typus: Mattfeldanthus mutisoides H. Rob. \& R. M. King

Arbustos erectos o apoyantes, con tallos simples, ramificados sólo en su parte superior. Hojas alternas, cortamente pecioladas, ovadas u ovado-lanceoladas. Inflorescencias cimosas, foliosas, con ramificaciones principales opuestas, flexuosas. Capítulos sésiles o brevemente pedunculados, solitarios, axilares, discoideos o disciformes. Involucro acampanado o cilíndrico, formado por 8-10 series de filarios imbricados, los exteriores gradualmente menores. Flores numerosas, cigomorfas, raramente actinomorfas en el centro del capítulo. Corola tubulosa, 5-lobada, lóbulos lanceolados, con espículas cortas en la porción apical, generalmente cigomorfa, con los cuatro lóbulos externos más cortos que el interno, raro actinomorfa en las flores centrales, con lóbulos de igual longitud. Anteras sagitadas, apéndice apical ovado-lanceolado o lanceolado. Estilo piloso, con ramas breves, lineares. Aquenios cilíndricos, 10-costados, seríceopubescentes. Papus blanco, biseriado, con la serie externa aplanada, corta, y la serie interna setácea. Polen tricolporado, equinolofado, de 50-60 $\mu \mathrm{m}$ diám., con muros discontinuos, microperforados. 


\section{Clave para diferenciar las especies de Mattfeldanthus}

1. Involucro anchamente acampanado, de 12-15 mm alt. Ramas de la inflorescencia 2-7-céfalas. Corolas de 12-16 mm long. Filarios lanceolados, todos acuminados.

1. M. andrade-limae (G. M. Barroso) Dematteis

1'. Involucro cilíndrico o estrechamente acampanado, de 24-28 mm alt. Ramas de la inflorescencia 1-3céfalas. Corolas de 18-22 mm long. Filarios ovados u oblongo-lanceolados, los internos redondeados u obtusos, los externos subagudos.

1. Mattfeldanthus andrade-limae (G. M. Barroso) Dematteis

Figs. 1 y 2

Dematteis, M., Taxon 52: 284. 2003.

Vernonia andrade-limae G. M. Barroso, Rodriguésia 35-36: 5. 1960-1961. Typus: Brasil. Pernambuco: Santa María da Bôa Vista, Fazenda Algodões, pequeno arbol subescandente, caule suberoso, 4-VII-1955, D. Andrade Lima 55-2119 (holotypus IPA!, isotypi CTES!, LP!, RB!).

Vernonia nobilis H. Rob., Phytologia 44 (4): 291. 1979. Typus: Brasil. Bahía: Vicinity of Machado Portello, 19-23-VI-1915, J. N. Rose \& P. G. Russel 19966 (holotypus US, isotypus NY, foto CTES!).

Mattfeldanthus nobilis (H. Rob.) H. Rob., Phytologia 45 (2): 166. 1980.

Arbusto erecto o raramente apoyante, de 1$1,8 \mathrm{~m}$ alt. Tallos estriados, glabros, castañovioláceos, laxamente pubérulos en los nudos, áfilos y cicatricosos en su parte inferior, redondeados y hojosos en la porción florífera. Hojas membranáceas, rugosas, cortamente pecioladas, con pecíolo de $2-5 \mathrm{~mm}$ long. Lámina ovada u ovado-lanceolada, subentera o irregularmente ondulada, aguda en el ápice, redondeada a subcordada en la base, glabra o escabrosa en el haz, laxamente pilosa sobre las venas principales, lanuginosa y punteadoglandulosa en el envés, pinnatinervada, venas secundarias 5-8, levemente arqueadas, de 4-6 $\mathrm{cm}$ long. x 2-3 cm lat. Inflorescencia cimosa, con ramas flexuosas, 2-7-céfalas, de $5-15 \mathrm{~cm}$ long. Capítulos discoideos, brevemente pedunculados, axilares, solitarios, separados en- tre sí por 10-18 mm. Pedicelos de 1-2 mm long., frecuentemente cubiertos por los filarios más externos. Brácteas de la inflorescencia foliáceas, siempre mayores que los capítulos. Involucro acampanado, de 12-15 mm alt. Filarios en 8-9 series, acuminados en el ápice, espiculados en el margen, castaños en el dorso, con el borde amarillento, los internos lanceolados, los externos ovado-lanceolados. Flores 35-40, violáceas o rojizas. Corola cigomorfa, de 12-16 mm long., lóbulos lanceolados, los cuatro externos de 3,2-4,4 $\mathrm{mm}$ long., el interno de 5-5,4 mm long., todos con espículas rígidas en el ápice. Anteras sagitadas, tecas de 4,5-5,2 $\mathrm{mm}$ long. Estilo de 13-15 mm long., ramas lineares, de 3,8-4,2 $\mathrm{mm}$ long. Aquenios densamente seríceos, de 2,5-3 mm long. Papus blanco, biseriado, la serie externa estrechamente lanceolada, de 1,5-2 mm long., la serie interna setácea, de 9$11 \mathrm{~mm}$ long.

Distribución geográfica y hábitat: Es una especie endémica del nordeste de Brasil, que ocupa el oeste del estado de Pernambuco y la región central de Bahía, dónde presenta una distribución bastante restringida. Habita casi siempre en la "caatinga", vegetación xerofítica exclusiva del nordeste de Brasil. Florece y fructifica durante otoño e invierno, generalmente entre abril y julio.

Material adicional estudiado: BRASIL. Bahía: Estação Ecológica Raso da Catarina, próximo a sede, 8-VII-1983, Queiroz 736 (HUEFS); Raso da Catarina, 16-V-1981, Bautista 457 (RB); Mun. Abaíra, Distrito de Catolés, Estrada CatolésAbaíra, ca. 4 a $5 \mathrm{~km}$ de Catolés, Engenho de 
M. Dematteis, Revisión de Mattfeldanthus (Asteraceae)

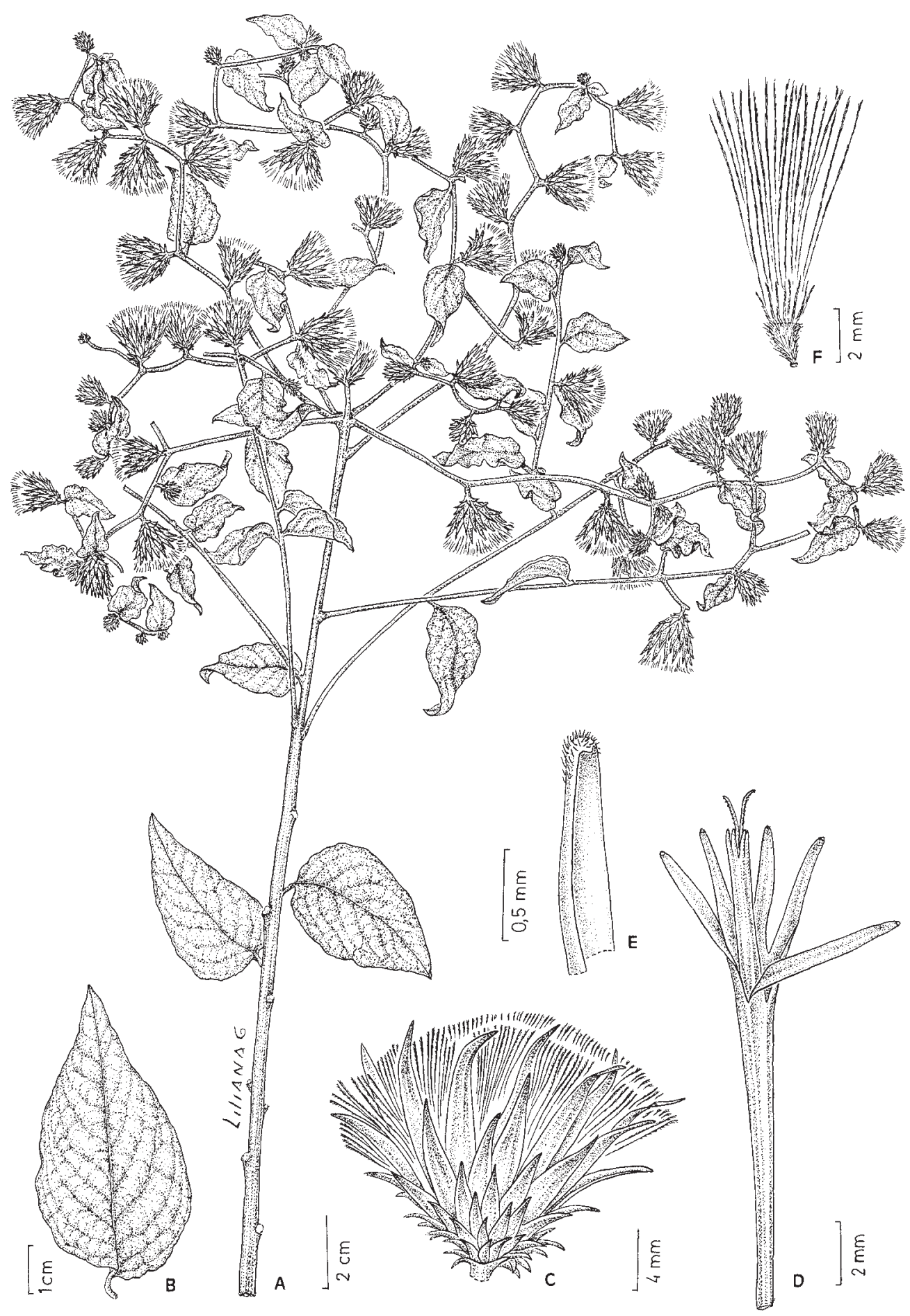

Fig. 1. Mattfeldanthus andrade-limae. A: rama florífera. B: hoja. C: capítulo. D: corola, con anteras y estilo. E: ápice del lóbulo de la corola. F: aquenio inmaduro con papus (A, Hatschbach \& al. 65167, CTES. B-E, Andrade Lima 552119 isotypus CTES). 


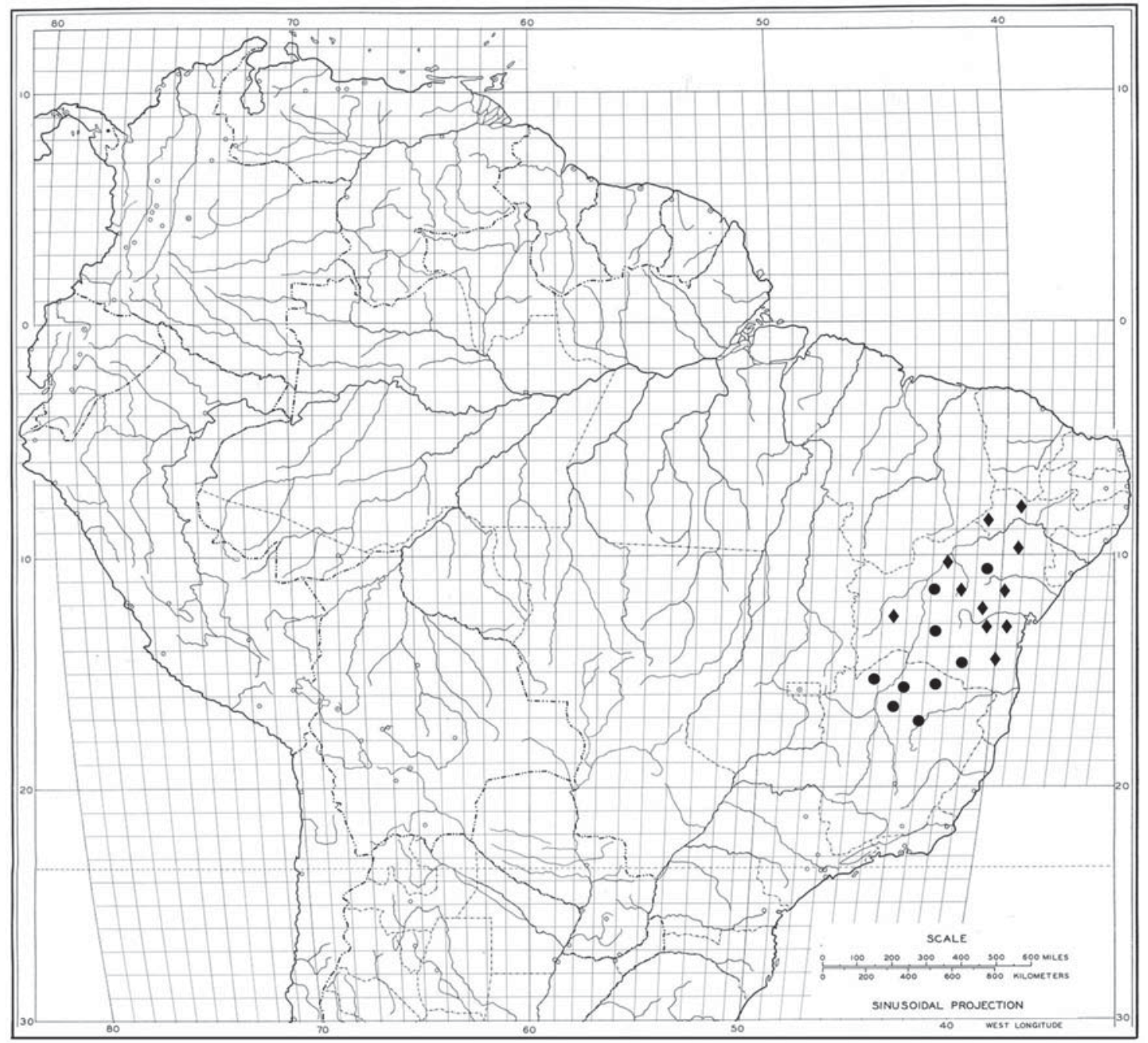

Fig. 2. Distribución geográfica de las dos especies de Mattfeldanthus. M. andrade-limae. $\bullet$ M. mutisoides.

Baixo, 19-V-1992, Ganev 316 (CTES, HUEFS, SPF); Mun. Abaíra, Engenho de Baixo, 9-VII1992, Ganev 635 (CTES, SPF); Mun. Abaíra, Próximo ao São José, 14-VI-1994, Ganev 3376 (SPF); Rod. BA-030, 10 km O de Brumado, 17VI-1986, Hatschbach \& Silva 50443 (C, MBM); Umburanas, Serra do Curral Feio, Cachoeirinha, a beira do rio Tabuleiro, ca. $10 \mathrm{~km} \mathrm{NW}$ de Delfino, 12-IV-1999, Queiroz \& al. 5444 (HUEFS); Mun. Caetité, Rodovia Piramirim a Caetité, próximo a Maniaçu, 21-IV-1996, Hatschbach \& al. 65167 (C, CTES, MBM); Mun. Iaçu, Rod. BA-046, Caatinga, 17-VII-1982, Hatschbach \& Guimarães 45096 (MBM); Mun. Iramaia, ca. 3 km do Pé de Serra na estrada para Contendas do Sincorá, 9-IV-
1999, Sant'Ana \& al. 734 (G, MBM, SP); Mun. Milagres, Tartaruga, 26-VI-2003, Hatschbach \& al. 75725 (CTES, MBM); Mun. Tremendal, km 79 da BA-262, trecho Anaje-Aracatú, 18-VII-1991, Sant'Ana \& al. 3 (MBM). Pernambuco: Afranio, margen da estrada, $2 \mathrm{~km}$ em direção ao Piauí, 22IV-1971, Acad. Bras. Ciên. 281 [Heringer, Andrade Lima, Sobrinho \& Sarmiento] (LP, R, RB); Santa María da Boa Vista, próximo a Lagoa, 3-V-1971, Acad. Bras. Ciên. 472 [Heringer, Andrade Lima, Sobrinho \& Sarmiento] (LP, R, RB).

Esta entidad presenta ciertas similitudes con M. mutisoides, pero puede distinguirse por la superficie de las hojas, el tamaño de la 
serie externa del papus y el número de flores, además de los caracteres indicados en la clave.

\section{Mattfeldanthus mutisoides H. Rob. \& R. M. King}

Figs. 2 y 3

Robinson, H. \& R. M. King, Willdenowia 9: 10. 1979. Typus: Brasil. Bahía: Cocos, Espigão Mestre, shrubby woods on hills of gentle slopes, ca. $13 \mathrm{~km}$ south of Cocos and $3 \mathrm{~km}$ south of the Rio Itaguarí, shrub 1,5 m tall, flowers brigth red, 15-III-1972, W. R. Anderson, M. Stieber \& J. H. Kirkbride 36985 (holotypus NY, foto CTES!, isotypus US, foto CTES!).

Arbusto erecto o apoyante, de 1-1,5 m alt. Tallos estriados, rojizos, glabros o laxamente pubérulos. Hojas membranáceas, planas, pecioladas, con pecíolo de 2-4 $\mathrm{mm}$ long. Lámina ovada, entera, obtusa en la base, aguda o acuminada en el ápice, escabrosa en el haz, albo-tomentosa en el envés, pinnatinervada, venas secundarias 5-6, suavemente arqueadas, de 3-6 cm long. x 2-3 cm lat. Inflorescencia cimosa, ramas flexuosas, 1-3-céfalas, de 10-15 cm long. Capítulos discoideos o disciformes, muy brevemente pedunculados o sésiles, axilares, solitarios, separados por 20 $50 \mathrm{~mm}$. Pedicelos de 1-3 mm long., cubiertos por los filarios más externos. Brácteas de la inflorescencia foliáceas, más largas que los capítulos. Involucro cilíndrico o muy estrechamente acampanado, 24-28 mm alt. Filarios en 8-10 series, espiculados en el margen, castaños en el dorso, amarillentos en el borde, los externos ovados, subagudos, los internos lanceolados u oblongo-lanceolados, redondeados u obtusos en el ápice. Flores anaranjadas o rojizas, 15-20, corola tubulosa, de 18-22 mm long., con espículas en el ápice de los lóbulos. Flores marginales con corola cigomorfa, lóbulos externos de 5-6 mm long., lóbulo interno de 8-9 mm long.; flores centrales iguales a las periféricas o con corola actinomorfa, todos los lóbulos de 8-9 mm long. Anteras sagitadas, tecas de 4-4,5 mm long. Estilo de 20-21 mm long., ramas lineares, de
3,8-4,3 mm long. Aquenios seríceos, de 2,8-3 $\mathrm{mm}$ long. Papus blanco, biseriado, serie externa linear, de 1-1,2 mm long., serie interna setácea, de 9-11 mm long.

Distribución geográfica y hábitat: Esta especie fue descripta originariamente sólo para el estado de Bahía. Sin embargo, colecciones recientes amplían su área de distribución también al norte de Minas Gerais, donde aparentemente es más frecuente que en Bahía. Habita en campos rupestres y en la "caatinga". Florece y fructifica en otoño e invierno, desde marzo hasta agosto.

Material adicional estudiado: BRASIL. Bahía.

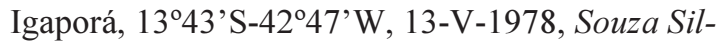
va 502 (RB, SP); Mun. Tanque Novo, Fazenda Boiada, 17-IV-2004, Hatschbach \& al. 77979 (MBM); Lagoa dos Patos, 27 km S de Irecê, 20VIII-1980, Socorro 96 (RB). Minas Gerais. Januária, Vale do Rio Peruaçu, Mirante no topo da quarta clarabóia do Janelão, 21-VII-1997, Salino \& Stehmann 3331 (BHCB); Mato Verde, São João do Bonito, estrada para Lavras na Serra Geral, 2,5 km de São João do Bonito, 7-IV-2004, Mello-Silva \& al. 2596 (SPF); Mun. Monte Azul, Serra do Espinhaço, 18-IV-1996, Hatschbach \& al. 64966 (C, CTES, MBM, RB, SPF); Mun. Montes Claros, Parque do Sapucaia, 15-IV-1990, Castro 17379 (BHCB, SPF).

En la descripción original, se consideró como uno de los caracteres principales para diferenciar al género la presencia de flores de dos tipos, las externas cigomorfas y las internas actinomorfas (Robinson \& King, 1979). Sin embargo, el material analizado de $M$. mutisoides en el presente estudio muestra todas las flores con corola cigomorfa, lo cual sugiere que podría tratarse de una variación intraespecífica.

El aspecto general de la planta se asemeja bastante a Vernonia laurifolia DC. y parte del material analizado de Mattfeldanthus mutisoides se encontraba bajo este nombre. Ambas especies se parecen en la forma de las hojas, el involucro y el tipo de ramificaciones, pero $V$. laurifolia tiene capítulos largamente pedunculados y menor número de series de filarios, además de presentar corola actinomorfa. 


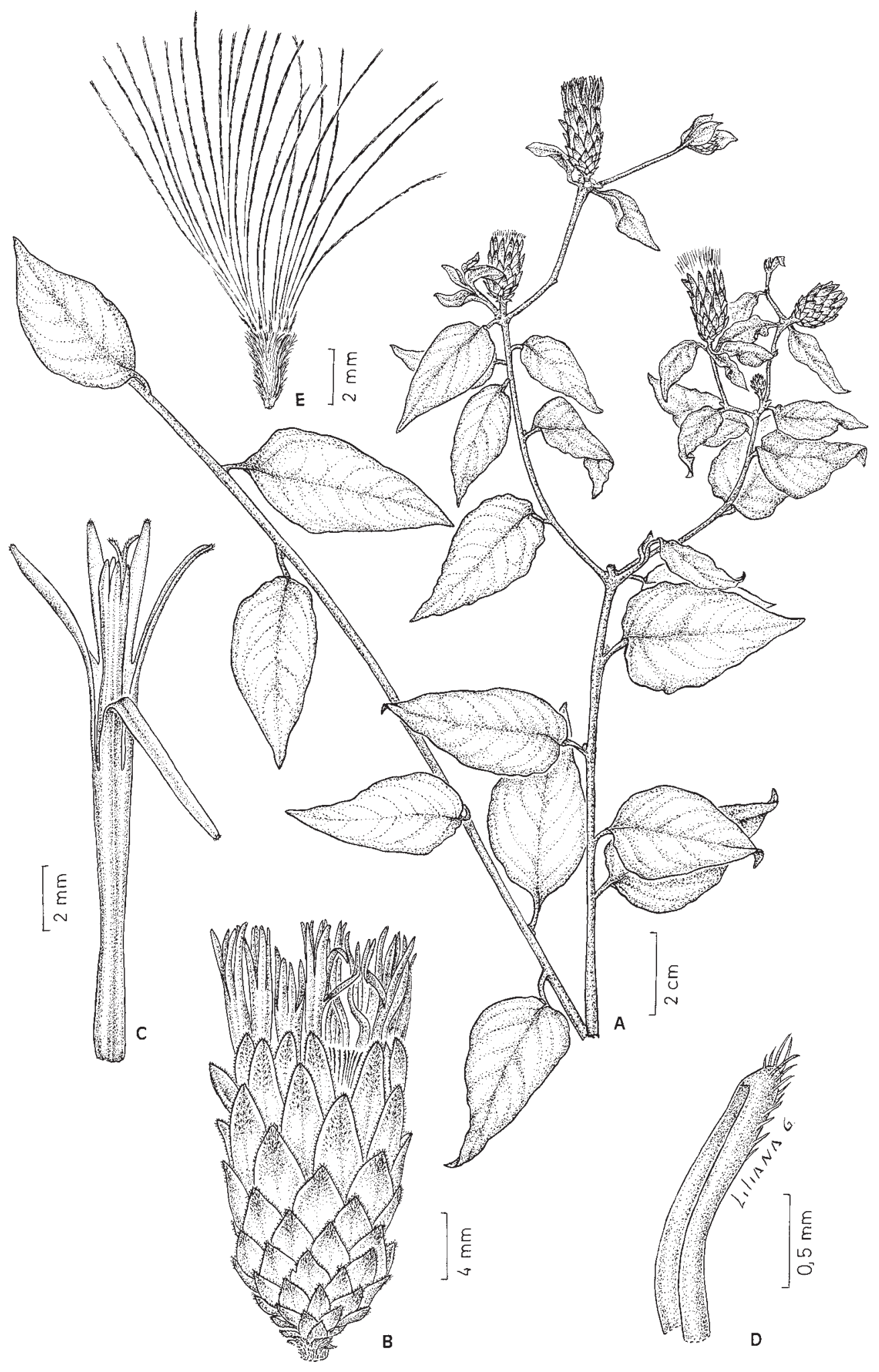

Fig. 3. Mattfeldanthus mutisoides. A: rama florífera. B: capítulo. C: corola, con anteras y estilo. D: porción apical de un lóbulo de la corola. E: aquenio con papus (A-D, Hatschbach \& al. 64966 CTES). 
También difiere de $M$. mutisoides, en la distribución geográfica y ecología, ya que $V$. laurifolia habita en selvas húmedas del oeste de Brasil y en las yungas de Bolivia.

\section{Agradecimientos}

Al personal y curadores de los herbarios consultados por su desinteresada colaboración y el préstamo de los ejemplares. Al Dr. José L. Panero de la Universidad de Texas por las valiosas sugerencias realizadas. A Mirta Liliana Gómez que preparó gentilmente las ilustraciones. Este trabajo se realizó mediante el apoyo del Consejo Nacional de Investigaciones Científicas y Técnicas (CONICET) y la Secretaría General de Ciencia y Técnica de la Universidad Nacional del Nordeste.

\section{Bibliografía}

BAKER, J. G. 1873. Compositae. I. Vernoniaceae. In C. Martius (ed.), Fl. bras. 6 (2): 1-179.

BARROSO, G. M. 1960-1961. Compositae novae. Rodriguésia 35-36: 5-9.

BENTHAM, G. 1873. Vernonieae. In G. Bentham \& J. D. Hooker (eds.), Gen. pl. 2 (1): 227-231.

BLAKE, S. F. 1945. Dipterocypsela, a new genus of Vernonieae from Colombia. J. Wash. Acad. Sci. 35(2): 36-38.

BREMER, K. 1994. Asteraceae. Cladistics and classification. Timber Press, Portland, Oregon.

CABRERA, A. L. 1944. Vernonieas Argentinas (Compositae). Darwiniana 6: 265-379.

DE CANDOLLE, A. P. 1836. Prodromus Systematis Naturalis Regni Vegetabilis, 5. Treuttel \& Würtz, Paris.

DEMATTEIS, M. 2002. Cytotaxonomic analysis of South American species of Vernonia (Vernonieae:
Asteraceae). Bot. J. Linn. Soc. 139 (4): 401-408.

- 2003. New species and new combinations in Brazilian Vernonieae (Asteraceae). Taxon 52: 281286.

HIND, D. J. N. 1993. Notes on the Compositae of Bahía, Brazil: I. Kew Bull. 48: 245-277.

JEFFREY, C. 1988. The Vernonieae in East tropical Africa. Notes on the Compositae V. Kew Bull. 43(2): 195-277.

JONES, S. B. 1977. Vernonieae - systematic review. In V. H. Heywood, J. B. Harborne \& B. L. Turner (eds.). The Biology and Chemistry of the Compositae. I. Academic Press, London.

- 1979. Synopsis and pollen morphology of Vernonia (Compositae: Vernonieae) in the New World. Rhodora 81: 425-447.

—_. 1981. Synoptic classification and pollen morphology of Vernonia (Compositae: Vernonieae) in the Old World. Rhodora 83: 59-75.

KEELEY, S. C. 1978. A revision of the West Indian Vernonias (Compositae). J. Arnold Arbor. 59: 360413.

— \& B. L. TURNER. 1990. A preliminary cladistic analysis of the genus Vernonia (Vernonieae: Asteraceae). Pl. Syst. Evol., Suppl. 4: 45-66.

LESSING, C. F. 1829. De Synanthereis herbarii regii berolinensis. Dissertatio prima. Linnaea 4: 420356.

ROBINSON, H. 1979. New species of the Vernonieae (Asteraceae). II. Five new species of Vernonia from Bahía. Phytologia 44 (4): 287-299.

_. 1980. New species of the Vernonieae (Asteraceae). V. Additions to Vernonia from Brazil. Phytologia 45 (2): 166-208.

- - 1988. Studies in the Lepidaploa complex (Vernonieae: Asteraceae). IV. The new genus Lessingianthus. Proc. Biol. Soc. Wash. 100: 929-951.

. 1999. Generic and subtribal classification of American Vernonieae. Smithsonian Contr. Bot. 89: 1-116.

— \& R. M. KING. 1979. Mattfeldanthus mutisoides gen. et spec. nov. (Asteraceae: Vernonieae) from Bahía, Brazil. Willdenowia 9: 9-12.

Original recibido el 1 de junio de 2005; aceptado el 30 de junio de 2005. 